\title{
Pre-kidney transplant lower extremity impairment and transplant length of stay: a time-to-discharge analysis of a prospective cohort study
}

Anthony J. Nastasi ${ }^{1,2 \dagger}$, Tyler S. Bryant ${ }^{1 \dagger}{ }^{\text {, Jimmy T. Le }}{ }^{1 \dagger}$, Jennifer Schrack ${ }^{1}$, Hao Ying ${ }^{2}$, Christine E. Haugen ${ }^{2}$, Marlís González Fernández ${ }^{3}$, Dorry L. Segev ${ }^{1,2}$ and Mara A. McAdams-DeMarco ${ }^{1,2^{*}}$ (D)

\begin{abstract}
Background: Few objective tests can be performed at admission for kidney transplantation $[\mathrm{KT}]$ to discern risk of increased length of stay [LOS], which is important for patient counseling and is associated with increased costs and mortality. The short physical performance battery [SPPB] is an easily administered, potentially modifiable, 3-part test of lower extremity function. SPPB score is associated with longer hospital LOS in older adults, and may provide similar utility in KT recipients given that ESRD is a disease of accelerated aging. The aim of this study was to characterize the association between SPPB-derived lower extremity function and LOS.
\end{abstract}

Methods: The SPPB was administered at KT admission in a prospective cohort of 595 recipients (8/2009-6/2016). The independent association between SPPB impairment (score $\leq 10)$ and LOS was tested with an adjusted conventional generalized gamma parametric survival model.

Results: Impaired recipients experienced longer LOS (median: 10 vs. 8 days; $P<0.001$ ) with the greatest difference in percent discharged on day 10 (impaired: 54.5\%, unimpaired: 73.3\%). Discharge typically took $13 \%$ longer in the impaired group (relative time $=1.13 ; 95 \% \mathrm{Cl}: 1.05,1.21, P=0.001$ ). Discharge for impaired recipients compared to unimpaired was least likely at day 5 (hazard ratio $=0.71 ; 95 \% \mathrm{Cl}: 0.68,0.74, P<0.001$ ). No differences in the SPPB impairment-LOS relationship were found by age (interaction $P=0.74$ ).

Conclusions: Pre-KT SPPB impairment was independently associated with longer LOS regardless of age, indicating that it is a useful, objective tool for pre-KT risk assessment in younger and older recipients that may help inform discharge planning.

Keywords: Kidney transplantation, Length of stay, Survival analysis

\section{Background}

Length of stay [LOS] is an important outcome associated with increased complications, death, and costs in transplant recipients [1-3]. A clear understanding of hospital LOS following kidney transplantation $[\mathrm{KT}]$ allows for

\footnotetext{
* Correspondence: mara@jhu.edu

${ }^{+}$Anthony J. Nastasi, Tyler S. Bryant and Jimmy T. Le contributed equally to this work.

'Department of Epidemiology, Johns Hopkins Bloomberg School of Public Health, 615 N. Wolfe St, Baltimore, MD 21205, USA

2Department of Surgery, Johns Hopkins School of Medicine, Baltimore, MD, USA

Full list of author information is available at the end of the article
}

appropriate informed consent, improved patient and caregiver counseling, as well as better discharge planning. We have recently identified transplant, recipient, and donor factors that are associated with LOS in KT recipients including pre-KT frailty [4]. Other than a frailty assessment [4-9], there is a dearth of inexpensive tests that can be performed successfully at admission for KT to identify recipients at risk of longer LOS [10].

The Short Physical Performance Battery [SPPB] is a well-validated, inexpensive, objective assessment tool of lower extremity function that was developed in community-dwelling older adults. Functional limitations, 
including SPPB impairment, have been shown to be associated with many important health outcomes including mortality, quality of life, functional decline, and LOS in older adults [11-14]. Our group recently found that SPPB impairment is associated with a 2-fold increased risk of post-KT mortality [15]. Given this strong association, it is likely that SPPB impairment is also associated with other KT outcomes, such as longer LOS. The SPPB measures the modifiable risk factor of functional limitations and may provide a target for interventions to prevent longer LOS.

However, there is currently no consensus regarding the most appropriate way to model the relationship between LOS and predictors of functional limitations such as the SPPB [16-18]. Conventional regression modeling treats LOS as a count of the number of days in the hospital, but does not appropriately account for patients who die during the hospitalization (non-informative censoring) and the skewed distributions of extended LOS, both of which are more common in older populations. Treating LOS as a time-to-discharge (i.e. time-to-event) analysis is seldom carried out when examining LOS, yet it allows for the calculation of informative, novel metrics of high utility for patient counseling and discharge planning [17]. This method allows for more clinically meaningful metrics to be calculated; for example, it provides the probability of being discharged each day for patients with and without SPPB impairment.

The goals of this study were to: (1) quantify the association between SPPB-derived lower extremity function and KT LOS, (2) stratify KT recipients by LOS, and (3) estimate novel and more clinically meaningful metrics of LOS by SPPB impairment using time-to-discharge analyses. We hypothesized that recipients with SPPB impairment at admission for KT would be at increased risk of longer LOS.

\section{Methods}

\section{Study design}

We studied $652 \mathrm{KT}$ recipients who were enrolled in a longitudinal cohort study at the Johns Hopkins Hospital, Baltimore, Maryland between August 2009 and June 2016. We excluded patients whose LOS was longer than 30 days post-KT as has been done previously due to methodological and generalizability concerns $(n=57)$, leaving 595 recipients in the analysis $[19,20]$. We abstracted LOS from medical records along with the recipient, transplant, and donor factors that were previously identified as potential risk factors for longer LOS [4]. Recipient factors include age, sex, race, education, body mass index, hepatitis $\mathrm{C}$ status, preemptive KT (i.e., KT before patient initiates dialysis), years on dialysis, previous $\mathrm{KT}$, and cause of end-stage renal disease (ESRD) including hypertension, diabetes, glomerulonephritis, or other cause. Additionally, we included recipient comorbidity (as measured by the Charlson Comorbidity Index [CCI] adapted for ESRD), which was both abstracted from the medical record and self-reported [21]. Transplant factors include panel reactive antibody, $\mathrm{ABO}$ incompatibility, human leukocyte antigen mismatches, cold ischemic time, and donor type (live vs. deceased). Donor factors include age, sex, race, donation after cardiac death, deceased expanded criteria, hypertension, diabetes, hepatitis $\mathrm{C}$ status, and creatinine. The Johns Hopkins Institutional Review Board approved this study (NA_00015758) and all participants provided written informed consent.

\section{Short physical performance battery (SPPB)}

The SPPB consists of 3 objective physical assessments (standing balance, walking speed, and repeated chair stands) of lower extremity function each with a score ranging from 0 to 4 with a summed composite score ranging from 0 to 12 , as has been previously described [11]. For the balance portion, recipients were asked to stand and remain in several progressively more difficult positions (side-by-side, semi-, and full-tandem stances) for $10 \mathrm{~s}$ each. If a position could not be held, no further positions were attempted. For the walking speed test, recipients' usual walking speed was timed from the first foot fall over the starting line to the first foot fall over the finish line of an 8 - $\mathrm{ft}$ course. Finally, for the chair stand portion, recipients were asked to fold their arms across their chest and rise from a chair 5 times as quickly as possible. The SPPB was measured at the time of hospital admission for KT, prior to the start of maintenance immunosuppressive medications and inpatient dialysis; a score of $\leq 10$ was decided a priori to signify impaired lower extremity function based on previous work looking at post-KT mortality [15].

\section{SPPB \& LOS}

We treated the LOS analysis as a time-to-event analysis and calculated the cumulative incidence of discharge using a Kaplan-Meier approach. This approach allows for a more detailed examination of discharge by impairment group with respect to time after KT and accounts for any non-informative censoring. Furthermore, using this approach we were able to calculate more clinically useful measures by estimating the changes in the relative hazard of discharge over time by SPPB impairment status as well as how many more days were needed for a given percent of impaired patients to be discharged compared to unimpaired.

We tested proportional hazards visually by graphing the $\log$-log plot of survival and statistically using Schoenfeld residuals $(P=0.04)$, indicating the likelihood of non-proportional hazards. Therefore, we explored several parametric survival models and selected the generalized gamma [GG] model [22] based on a comparison of log likelihoods and Akaike Information Criteria. This model had the highest model quality, balancing fit and 
parsimony as has been previously demonstrated in LOS modeling [17]. For the GG model, we estimated 3 parameters, $\beta$ (a location parameter that is related to median time to discharge), $\sigma$ (a dispersion or scale parameter related to the interquartile ratio: third quartile divided by the first quartile of lengths of stay), and $\mathrm{\kappa}$ (shape). We modeled the relative hazard with time and the relative time to discharge, the factor by which times were expanded or contracted. For example, a relative time of 2 would mean that it generally took twice as long for SPPB impaired recipients to be discharged compared to the unimpaired recipients and would correspond with a relative hazard $<1$.

We quantified the association between SPPB impairment and LOS after adjusting for age, sex, race, body mass index, years on dialysis, cause of ESRD, and donor type. These covariates were selected based on their relevance to LOS as well as their use in previous literature [4]. To assess the fit of this model, we graphically overlaid the GG cumulative discharge functions with the non-parametric KaplanMeier cumulative discharge functions for comparison. Death was not treated as a competing risk as only 2 patients died during hospitalization.

\section{SPPB \& LOS $\geq 14$ days}

In addition, we used logistic regression models to assess both the relationship between SPPB impairment and LOS $\geq 14$ days adjusting for the same factors as in the GG model. LOS $\geq 14$ days was selected as an a priori outcome because it is seen as clinically relevant and has been previously used as a threshold for longer LOS after KT [4]. Using a similar approach, we assessed the independent association between a 1-point decrease in SPPB score (worse function) as well as each individual component of the SPPB (balance, walking speed, and chair stands) and LOS $\geq 14$ days.

\section{Effect heterogeneity}

Effect modification of the associations between SPPB impairment and LOS $\geq 14$ days by recipient age, sex, race, diabetes status, and frailty status were tested using a Wald test. We used a similar approach to test for effect modification between as well as SPPB composite score and LOS $\geq 14$ days.

\section{Sensitivity analysis of SPPB and LOS}

We assessed the robustness of the adjusted associations between SPPB performance (composite score, impairment) and LOS (logistic model with LOS $\geq 14$ days, GG model with continuous LOS) by including KT recipients omitted previously from the primary analysis for having a LOS > 30 days due to generalizability concerns (i.e., these patients not representing the typical KT recipient) as well as additionally adjusting for comorbidities including cardiovascular disease (including any ischemic heart disease, cerebrovascular disease, or peripheral vascular disease), lung disease, diabetes status, and CCI adapted for ESRD.

\section{Statistical analysis}

A $P$ value $<0.05$ was considered statistically significant. All analyses were performed using Stata (version 14; StataCorp, College Station, TX) and R Statistical software version 3.3 (http://www.r-project.org).

\section{Results}

\section{Study population}

The mean age of the $595 \mathrm{KT}$ recipients was 51.8 years (SD =14.1, range: 18.7-86.0); 225 (37.8\%) were female, 255 (42.9\%) were African American, and 212 (35.6\%) were live donor recipients. At time of $\mathrm{KT}, 47.1 \%$ of $\mathrm{KT}$ recipients were SPPB impaired. The median LOS was 8 days (IQR: 713, range: $2-30)$. Tacrolimus was associated with a shorter LOS $(P=0.02)$; no other association between immunosuppressive medication (thymoglobulin, mycophenolate mofetil, or prednisone) and LOS was found.

\section{SPPB at the time of admission for KT}

The median SPPB score for the $595 \mathrm{KT}$ recipients was 11 (IQR: 9-12, range 0-12). On average, impaired recipients were significantly older (56.3 vs. 47.8 years; $P<0.001$ ), had higher body mass indices ( 28.1 vs. $26.7 ; P=0.004$ ), were on dialysis for a longer duration (3.3 vs. 2.7 years; $P=0.01$ ), were more likely to be black $(P=0.01)$ and have diabetes as their cause of ESRD $(P<0.001)$ compared to those who were unimpaired (Table 1). SPPB impaired individuals were more likely to have an expanded criteria donor $(11.1 \%$ vs. $4.0 \% ; P=0.01)$, a donor with hypertension $(21.8 \%$ vs. $13.0 \% ; P=0.02$ ), and a donor with a creatinine $>1.5 \mathrm{mg} / \mathrm{dL}$ (27.5\% vs. $20.0 \% ; P=0.03$ ). No association was found between SPPB impairment and immunosuppressive medication (tacrolimus, thymoglobulin, mycophenolate mofetil, or prednisone; all $P>0.35$ ).

\section{SPPB \& LOS}

SPPB impaired recipients experienced a longer LOS compared to unimpaired recipients $(P<0.001)$ (Fig. 1) such that impaired recipients had a median LOS of 10 days (IQR; 7-14) while unimpaired recipients had a median LOS of 8 days (IQR; 6-11). From the KaplanMeier calculations of cumulative discharge, by 7 days post-KT, $43.2 \%$ of unimpaired and $34.1 \%$ of impaired recipients were discharged (Fig. 1, Table 2). By 14 days post-KT, $87.9 \%$ of unimpaired and $75.3 \%$ of impaired recipients were discharged. By 21 days, 97.1\% of unimpaired and $92.8 \%$ of impaired recipients were discharged. The greatest difference in percent discharged between impaired and unimpaired recipients occurred 10 days post-KT at which time $18.8 \%$ more unimpaired recipients were discharged (Table 2). The conventional GG 
Table 1 Baseline Characteristics of Kidney Transplant Recipients by Short Physical Performance Battery (SPPB) Impairment Status, Baltimore, Maryland, 2009-2016

\begin{tabular}{|c|c|c|c|}
\hline Factors & $\begin{array}{l}\text { Unimpaired } \\
\text { (SPPB Score > 10) } \\
N=315\end{array}$ & $\begin{array}{l}\text { Impaired } \\
(\text { SPPB Score } \leq 10) \\
N=280\end{array}$ & $P$ Value \\
\hline \multicolumn{4}{|l|}{ Recipient factors } \\
\hline Age (years) & $47.8(14.1)$ & $56.3(12.6)$ & $<0.001$ \\
\hline Female & 39.4 & 36.1 & 0.41 \\
\hline Race & & & 0.01 \\
\hline White & 55.2 & 41.1 & \\
\hline Black & 36.2 & 50.4 & \\
\hline Other & 8.5 & 8.8 & \\
\hline Education & & & 0.06 \\
\hline High school (9-12) or less & 40.6 & 47.0 & \\
\hline 2-year technical school & 5.5 & 9.7 & \\
\hline College/technical school & 31.5 & 23.9 & \\
\hline Post-college graduate degree & 22.4 & 19.4 & \\
\hline Body mass index $\left(\mathrm{kg} / \mathrm{m}^{2}\right)$ & $26.7(5.1)$ & $28.1(6.2)$ & $<0.01$ \\
\hline Hepatitis C virus positive & 7.0 & 7.6 & 0.8 \\
\hline Preemptive transplant & 20.3 & 18.2 & 0.52 \\
\hline Years on dialysis $^{\mathrm{a}}$ & $1.7(0.1-3.9)$ & $2.4(0.4-4.8)$ & 0.01 \\
\hline Previous KT & 20.3 & 15.4 & 0.12 \\
\hline Cause of end-stage renal disease & & & $<0.001$ \\
\hline Hypertension & 30.8 & 34.3 & \\
\hline Diabetes & 10.5 & 21.1 & \\
\hline Glomerulonephritis & 2.9 & 5.4 & \\
\hline Other & 55.9 & 39.3 & \\
\hline \multicolumn{4}{|l|}{ Transplant factors } \\
\hline 0 Human leukoctye antigen mismatches & $15.1(14.0)$ & $22.0(15.5)$ & $<0.01$ \\
\hline Cold ischemic time $>24 \mathrm{~h}$ & 32.7 & 51.43 & $<0.001$ \\
\hline Live donor & 44.1 & 26.1 & $<0.001$ \\
\hline \multicolumn{4}{|l|}{ Donor factors } \\
\hline Age (years) & $37.7(13.9)$ & $38.5(15.4)$ & 0.36 \\
\hline Female sex & 49.5 & 43.9 & 0.17 \\
\hline Race & & & 0.42 \\
\hline White & 73.7 & 68.6 & \\
\hline Black & 17.5 & 23.2 & \\
\hline Other & 8.8 & 8.2 & \\
\hline Donation after cardiac death & 19.4 & 22.2 & 0.5 \\
\hline Expanded criteria donor & 4 & 11.1 & 0.01 \\
\hline Charlson Comorbidity Index ${ }^{a}$ & $0(0-2)$ & $1(0-3)$ & $<0.001$ \\
\hline
\end{tabular}

Percentages and mean (SD) are presented unless otherwise noted

${ }^{\mathrm{a}}$ Median and IQR are presented

models for impaired $(\beta=1.87, \sigma=0.41, \kappa=-0.56)$ and unimpaired $(\beta=1.75, \quad \sigma=0.41, \quad \kappa=-0.56)$ recipients closely mirrored the Kaplan-Meier cumulative discharge curves (Table 3, Fig. 1), confirming a good fit to describe the SPPB impairment-LOS relationship.
The adjusted hazard of discharge was lower for impaired recipients compared to unimpaired recipients until approximately 21 days post-KT as shown by an adjusted relative hazard $<1$ during this time (Fig. $2 a$ ). By day 6 , the adjusted relative hazard of discharge comparing impaired 


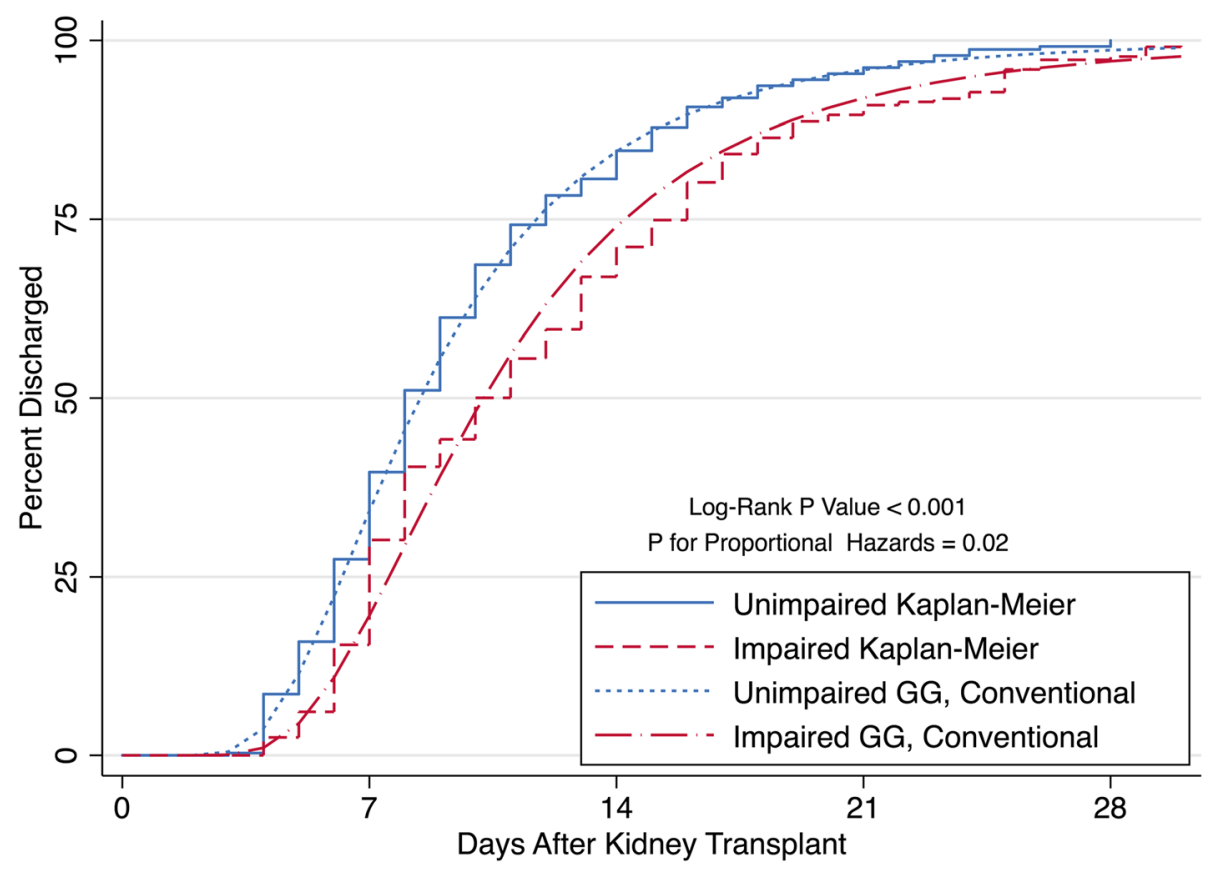

Fig. 1 Cumulative Percent Discharged by Short Physical Performance Battery (SPPB) Impairment Status Among Kidney Transplant Recipients $(n=595)$. SPPB impairment was defined as an SPPB score $\leq 10$. The Kaplan-Meier and conventional Generalized Gamma (GG) cumulative incidences of discharge are shown

to unimpaired recipients was at its lowest at 0.71 (95\% confidence interval (CI): 0.68, 0.74; $P<0.001$ ), indicating that impaired recipients had a $29 \%$ lower chance of being discharged on this day. The adjusted relative time comparing impaired to unimpaired recipients was 1.13 (95\% CI: 1.05, 1.21; $P=0.001)$, indicating that for the same percentage of recipients to be discharged, it typically took $13 \%$ longer in the impaired group compared to the unimpaired group (Table 3). Similarly, it took approximately 1 day, 2 days, and 3 days longer for impaired recipients to achieve 25, 50, and 75\% discharged compared to unimpaired recipients, respectively (Fig. 2b).

\section{SPPB \& LOS $\geq 14$ Days}

SPPB impaired recipients experienced an adjusted 1.90-fold (95\% CI: 1.23, 2.94; $P=0.004$ ) higher odds of a LOS $\geq 14$ days (Table 3). A 1-point decrease (worse function) in SPPB composite score was associated with an adjusted 1.16-fold (95\% CI: 1.08, 1.27; $P<0.001$ ) higher odds of a LOS $\geq 14$ days (Table 3 ). Each 1-point decrease (worse function) in the balance, walking speed, and chair stand component scores were associated with 1.54-fold (95\% CI: 1.27, 1.89; $P<$ 0.001), 1.33-fold (95\% CI: 1.08, 1.67; $P=0.01$ ), and 1.23-fold (95\% CI: 1.05, 1.45; $P=0.01$ ) higher odds of a LOS $\geq 14$ days, respectively.

\section{Effect heterogeneity}

No differences in the association between SPPB impairment status and LOS $\geq 14$ days were found by age (interaction $P=0.74$ ), race (interaction $P=0.34$ ), sex (interaction $P=0.20$ ), diabetes status (interaction $P=0.71$ ), or frailty status (interaction $P=0.20$ ). Similarly, no differences in the association between SPPB composite score and LOS $\geq 14$ days were found by age (interaction $P=0.67$ ), race (interaction $P=0.23$ ), sex (interaction $P=0.99$ ), diabetes status (interaction $P=0.63$ ), or frailty status (interaction $P=0.24)$.

\section{Sensitivity analysis of SPPB and LOS}

SPPB impairment and composite score remained significantly associated with LOS $\geq 14$ days in the fully adjusted logistic models that additionally adjusted for comorbidities including cardiovascular disease (including any ischemic heart disease, cerebrovascular disease, or peripheral vascular disease), lung disease, diabetes status, and CCI adapted for ESRD and included KT recipients with LOS > 30 days. Specifically, impairment was associated with a 1.74-fold (95\% CI: $1.31,2.65 ; P=0.01$ ) increase in risk of LOS $\geq 14$ days; a one-point decrease in SPPB score was associated with a 1.19 -fold (95\% CI: 1.08, 1.30; $P<0.001$ ) increase in risk. SPPB impairment also remained significantly associated with LOS in the conventional GG model such that the adjusted relative time comparing impaired to unimpaired recipients was 1.11 (95\% CI: 1.03, 1.19; $P=0.005$ ). 
Table 2 Cumulative Percent of Kidney Transplant Recipients Discharged by Short Physical Performance Battery (SPPB) Impairment Status, Baltimore, Maryland, 2009-2016

\begin{tabular}{|c|c|c|}
\hline Days Post-KT & SPPB Unimpaired ${ }^{\mathrm{a}}(\%)$ & SPPB Impaired (\%) \\
\hline 1 & 0 & 0 \\
\hline 2 & 0 & 0 \\
\hline 3 & 0.3 & 0 \\
\hline 4 & 9.2 & 2.5 \\
\hline 5 & 16.8 & 6.8 \\
\hline 6 & 29.8 & 17.2 \\
\hline 7 & 43.2 & 34.1 \\
\hline 8 & 55.6 & 44.4 \\
\hline 9 & 65.1 & 48.4 \\
\hline 10 & 73.3 & 54.5 \\
\hline 11 & 78.1 & 60.6 \\
\hline 12 & 82.2 & 64.5 \\
\hline 13 & 84.4 & 71.7 \\
\hline 14 & 87.9 & 75.3 \\
\hline 15 & 90.5 & 79.6 \\
\hline 16 & 93.0 & 83.9 \\
\hline 17 & 94.0 & 87.5 \\
\hline 18 & 95.2 & 89.3 \\
\hline 19 & 95.9 & 91.0 \\
\hline 20 & 96.5 & 91.8 \\
\hline 21 & 97.1 & 92.8 \\
\hline 22 & 97.8 & 93.2 \\
\hline 23 & 98.4 & 93.6 \\
\hline 24 & 99.1 & 94.3 \\
\hline 25 & 99.1 & 96.8 \\
\hline 26 & 99.4 & 97.9 \\
\hline 27 & 99.4 & 97.9 \\
\hline 28 & 100 & 98.2 \\
\hline 29 & 100 & 99.3 \\
\hline 30 & 100 & 100 \\
\hline
\end{tabular}

Abbreviations: KT Kidney Transplant, SPPB Short Physical Performance Battery ${ }^{\text {a }}$ SPPB impairment was defined as an SPPB score $\leq 10$

\section{Discussion}

In a prospective, longitudinal cohort study of $595 \mathrm{KT}$ recipients, we found that pre-KT SPPB composite score and lower extremity functional impairment were independently associated with increased LOS. Physical functioning in ESRD patients is important, given that ESRD is a disease of accelerated aging [10]. By modeling the relationship between SPPB impairment and LOS with a conventional GG model, we estimated that it took $13 \%$ longer for SPPB impaired recipients to be discharged compared to unimpaired recipients, and that impaired recipients experienced a consistently and
Table 3 Independent Associations of Short Physical Performance Battery Impairment with Kidney Transplant Length of Stay, Baltimore, Maryland, 2009-2016

\begin{tabular}{|c|c|c|}
\hline \multirow{3}{*}{$\begin{array}{l}\text { Model }^{\mathrm{a}} \\
\text { Conventional Generalized } \\
\text { Gamma (Time-to-Discharge) }\end{array}$} & \multicolumn{2}{|c|}{ Association between SPPB and LOS } \\
\hline & \multicolumn{2}{|l|}{ Relative Time $(95 \% \mathrm{Cl})$} \\
\hline & Impaired Vs. Unimpaired ${ }^{\mathrm{b}}$ & $1.13(1.05,1.21)$ \\
\hline Logistic Regression & \multicolumn{2}{|l|}{ Odds Ratio (95\% Cl) } \\
\hline \multirow[t]{2}{*}{ (LOS $\geq 14$ Days) } & Impaired Vs. Unimpaired & $1.90(1.23,2.94)$ \\
\hline & $\begin{array}{l}\text { One-point decrease in } \\
\text { SPPB composite score }\end{array}$ & $1.16(1.08,1.27)$ \\
\hline
\end{tabular}

Abbreviations: Cl Confidence Interval, LOS Length of Stay, SE Standard Error, SPPB Short Physical Performance Battery

${ }^{a}$ Adjusted for age, sex, race, body mass index, years on dialysis, cause of endstage renal disease, and donor type. GG model parameters were $\beta=1.87, \sigma=$ $0.41, \mathrm{~K}=-0.56$ and $\beta=1.75, \sigma=0.41, \mathrm{~K}=-0.56$ for the impaired and unimpaired recipients, respectively. Parameters for the GG model define the shape of the curve; $\beta$ (a location parameter that is related to median time to discharge), $\sigma$ (a dispersion or scale parameter related to the interquartile ratio: third quartile divided by the first quartile of lengths of stay), and $\mathrm{K}$ (shape)

${ }^{b}$ SPPB impairment was defined as an SPPB score $\leq 10$

significantly lower chance of being discharged until approximately 21 days post-KT. The component of the SPPB with the strongest association with LOS was the balance test in which a one-point decrease in balance score was associated with a 1.54-fold increase in odds of LOS $\geq 14$ days. These strong, independent, and consistent findings remained in both older and younger adults, highlighting the fact that ESRD is a disease of accelerated aging with outcomes strongly tied to function abilities. Our work also demonstrates the SPPB's great potential utility as a risk stratification tool in $\mathrm{KT}$, making it one of a limited selection of tools with such potential particularly among older surgical patients.

Frailty, an aging-related syndrome marked by diminished physiologic reserve, has been studied in ESRD patients and is the main assessment tool for risk stratification in transplantation [4-9, 23-30]. Frailty is also a strong predictor of important transplant outcomes including quality of life, LOS, early hospital readmission, and mortality $[4-7,9,23$, 29-32]. The SPPB, a measure of functional limitation, consists of completely objective components and is less burdensome to measure than frailty given that it consists of fewer components and requires no additional tools. These qualities facilitate its use in non-research, clinical settings, permitting improved patient counseling and other clinical goals for which frailty may not be as easily administered. Furthermore, transplant centers tend to have their own procedures for evaluation, waitlisting, and pre-operative counseling, often relying on comorbidities and age. The SPPB, therefore, offers a standard, reliable tool across centers.

Functional limitation as measured by SPPB has been found to be predictive of important health outcomes in a variety of populations [11, 33]. To our knowledge, three other works have looked at the SPPB in transplant 

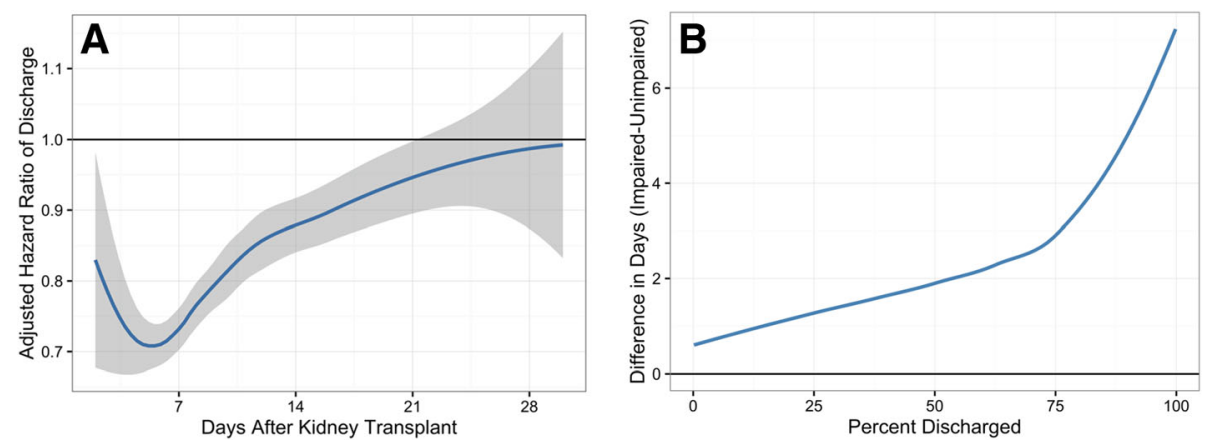

Fig. 2 a Relative Hazard (HR) of length of stay (LOS) over time, comparing kidney transplant (KT) recipients who are Short Physical Performance Battery (SPPB) impaired versus those who are not. The SPPB-impaired group has a significantly lower hazard of discharge compared to the unimpaired group until around day 21, when there is no longer a significant difference between the hazards of the two groups. $\mathbf{b}$ The number of days longer that SPPB impaired kidney transplant recipients needed to reach the same percentage discharged as the SPPB unimpaired recipients. Patients who are SPPB-impaired stay in the hospital longer regardless of the percentage of patients discharged in each group. The time to reach 50\% discharge was 2 days longer in the impaired group. Lower extremity impairment was defined as an SPPB score $\leq 10$

populations. Nastasi et al. and Wang et al. found an association between pre-KT or pre-liver transplant SPPB score, respectively, and waitlist death/delisting [34]. Lorenz et al. also found an association between pre-KT SPPB and LOS; however, this association was not explored in depth and was limited to a logistic regression of LOS $>4$ days [35]. Our findings extended this work to KT recipients in demonstrating a robust association between SPPB-measured lower extremity impairment and post-KT LOS.

Functional limitations is associated with worse outcomes in patients across the kidney disease spectrum. Longitudinal studies in aging have shown that functional limitation is a critical step in the pathway describing progression from disease to disability [36, 37]. This has been observe in patients with ESRD, where the trajectory of recovery is rapidly downward without efforts to improve or preserve physical functioning. For example, of the 366 patients who received a transplant within 24 months of initiating dialysis in the Dialysis Morbidity and Mortality Study, those with a lower self-reported physical functioning before KT were more likely to be rehospitalized after transplantation [38].

Functional limitation, however, can be modified through prehabilitiation. There is evidence that patients with higher physical functional capabilities before a surgical intervention will better tolerate a procedure such as KT. For example, a recent review of the literature by Cheng et al. identified a growing body of evidence documenting improved outcomes in cardiopulmonary fitness with exercise training in patients after cardiothoracic and orthopedic surgeries, including post-cardiac transplantation [39]. There is also evidence that most nephrologists, geriatricians, transplant surgeons, and ESRD patients agree that prehabilitation could make ESRD patients less frail [40]. Because functional limitations can be improved through intervention, pre-KT risk assessment with the SPPB may provide an opportunity to intervene by encouraging patient participation in a prehabilitation program.

To our knowledge, this was the first use of a parametric GG survival model to analyze LOS in a surgical population and to calculate novel LOS metrics, which provide a detailed and informative characterization of the differing trajectories of recipients with functional limitations over the course of hospital stay. These methods, in addition to being informative regarding clinical utility, appear to be the most appropriate methodologically when considering predictive ability and model quality $[16,17]$. These are likely even more important methodologic weaknesses to consider when looking at older surgical populations because they take into account noninformative censoring (e.g., death) and better handle skewed distributions of extended LOS, issues likely more common in older adults. Using our methodologically appropriate approach, a recipient can know their absolute and relative chance of discharge on any given day post-KT, which provides the chance to better inform care of older adult surgical patients.

A limitation of our work is that we only had data from a single transplant center, which may limit generalizability of our findings. However, we believe the participants in this study are at least representative of the entire KT population at our institution as our cohort of participating KT recipients were not significantly different from those that did not agree to participate and likely represent the $\mathrm{KT}$ population in general. Additionally, we found no effect heterogeneity by major recipient characteristics, further suggesting that our findings are generalizable to other populations that might not share the same characteristics as ours.

This study has several important strengths including its prospective design, the representativeness of the cohort, and the measurement of the SPPB, a novel measure of functional limitation. Ascertainment and assessment of recipient factors in this cohort are strengths given that the 
national registry data of KT recipients lack granular measurements of specific gerontology concepts like the SPPB.

\section{Conclusions}

Pre-KT SPPB impairment and composite score were both independently associated with increased LOS, suggesting that the SPPB is a high-utility objective physical assessment capable of successful post-KT risk stratification for LOS and potentially other important KT outcomes. Furthermore, the use of a parametric survival model like the GG model allowed for a more informative and methodologically appropriate analysis of LOS and should thus be considered in place of conventional regression approaches especially in older adult surgical populations given their unique methodologic considerations. Based on these findings, SPPB impaired individuals should be identified at time of KT to facilitate successful patient discharge counseling and to potentially receive more careful follow-up to avoid adverse events associated with an increased LOS.

\section{Abbreviations}

Cl: Confidence interval; ESRD: End-stage renal disease; GG: Generalized gamma; KT: Kidney transplantation; LOS: Length of stay; SPPB: Short Physical Performance Battery

\section{Acknowledgements}

A big "thank you" to Dr. Alvaro Muñoz, Dr. Christopher Cox, and the rest of the AMDACS team for their enlightening instruction and penetrating insight during the course of this work.

\section{Funding}

This work was supported by the National Institutes of Health [grant numbers R01 AG042504, K24 DK101828 to D.L.S.]; the National Institute on Aging and Johns Hopkins University Claude D. Pepper Older Americans Independence Center [grant number P30 AG021334 to M.M.D.]; and the National Institute on Aging [grant number R01 AG055781 to M.M.D.; K01 AG043501 to M.M.D., grant number K01 AG048765 to J.S., grant number T32 AG000247 to J.T.L., grant number F32 AG053025 to C.E.H.]. The funding bodies had no role in: 1) the design of the study, 2) the collection, analysis, and interpretation of the data or 3) the writing of the manuscript.

\section{Availability of data and materials}

The data are available by request and approval from the PI (Dr. Segev).

\begin{abstract}
Authors' contributions
All authors have made substantial contributions to conception and design, or acquisition of data, or analysis and interpretation of data; been involved in drafting the manuscript or revising it critically for important intellectual content; read and given final approval of the version to be published. Each author participated sufficiently in the work to take public responsibility for appropriate portions of the content and agreed to be accountable for all aspects of the work in ensuring that questions related to the accuracy or integrity of any part of the work are appropriately investigated and resolved. Specifically, the authors contributed to: Data Acquisition (AJN, TSB, JTL, DLS, MMD). Study Design (AJN, TSB, JTL, JS, HY, CEH, MGF, DLS, MMD). Statistical Analysis (AJN, TSB, JTL, HY, MMD).
\end{abstract}

Ethics approval and consent to participate

The Johns Hopkins Institutional Review Board approved this study (NA_00015758) and all participants provided written informed consent.

Consent for publication

Not applicable.

\section{Competing interests}

DLS reports receiving speaking honoraria from Sanofi, Genzyme, and CSL Behring. The other authors declare that they have no sources of funding or conflicts of interest relevant to the analysis to report.

\section{Publisher's Note}

Springer Nature remains neutral with regard to jurisdictional claims in published maps and institutional affiliations.

\section{Author details}

'Department of Epidemiology, Johns Hopkins Bloomberg School of Public Health, 615 N. Wolfe St, Baltimore, MD 21205, USA. ²Department of Surgery, Johns Hopkins School of Medicine, Baltimore, MD, USA. ${ }^{3}$ Department of Physical Medicine and Rehabilitation, Johns Hopkins University School of Medicine, Baltimore, MD, USA.

Received: 17 August 2017 Accepted: 9 October 2018

Published online: 19 October 2018

\section{References}

1. Banga A, Mohanka M, Mullins J, Bollineni S, Kaza V, Ring S, Bajona P, Peltz M, Wait $M$, Torres F. Hospital length of stay after lung transplantation: independent predictors and association with early and late survival. J Heart Lung Transplant.

2. Smith JO, Shiffman ML, Behnke M, Stravitz RT, Luketic VA, Sanyal AJ, Heuman DM, Fisher RA, Cotterell AH, Maluf DG, et al. Incidence of prolonged length of stay after orthotopic liver transplantation and its influence on outcomes. Liver Transpl. 2009;15(3):273-9.

3. Villa M, Siskind E, Sameyah E, Alex A, Blum M, Tyrell R, Fana M, Mishler M, Godwin A, Kuncewitch M, et al. Shortened length of stay improves financial outcomes in living donor kidney transplantation. Int J Angiol. 2013;22(2):101-4.

4. McAdams-DeMarco MA, King EA, Luo X, Haugen C, DiBrito S, Shaffer A, Kucirka LM, Desai NM, Dagher NN, Lonze BE, et al. Frailty, length of stay, and mortality in kidney transplant recipients: a National Registry and prospective cohort study. Ann Surg. 2016.

5. McAdams-DeMarco MA, Law A, King E, Orandi B, Salter M, Gupta N, Chow E, Alachkar N, Desai N, Varadhan R, et al. Frailty and mortality in kidney transplant recipients. Am J Transplant. 2015;15(1):149-54.

6. McAdams-DeMarco MA, Law A, Salter ML, Chow E, Grams M, Walston J, Segev DL. Frailty and early hospital readmission after kidney transplantation. Am J Transplant. 2013;13(8):2091-5.

7. McAdams-DeMarco MA, Law A, Tan J, Delp C, King EA, Orandi B, Salter M, Alachkar N, Desai N, Grams M, et al. Frailty, mycophenolate reduction, and graft loss in kidney transplant recipients. Transplantation. 2015;99(4):805-10.

8. McAdams-DeMarco MA, Law A, Salter ML, Boyarsky B, Gimenez L, Jaar BG, Walston JD, Segev DL. Frailty as a novel predictor of mortality and hospitalization in individuals of all ages undergoing hemodialysis. J Am Geriatr Soc. 2013;61(6):896-901.

9. Garonzik-Wang JM, Govindan P, Grinnan JW, et al. Frailty and delayed graft function in kidney transplant recipients. Arch Surg. 2012;147(2):190-3.

10. Johansen $\mathrm{KL}$, Chertow GM, Jin C, Kutner NG. Significance of frailty among Dialysis patients. J Am Soc Nephrol. 2007;18(11):2960-7.

11. Guralnik JM, Simonsick EM, Ferrucci L, Glynn RJ, Berkman LF, Blazer DG, Scherr PA, Wallace RB. A short physical performance battery assessing lower extremity function: association with self-reported disability and prediction of mortality and nursing home admission. J Gerontol. 1994;49(2):M85-94.

12. Oh B, Cho B, Choi H-C, Son K-Y, Park SM, Chun S, Cho S-I. The influence of lower-extremity function in elderly individuals' quality of life (QOL): an analysis of the correlation between SPPB and EQ-5D. Arch Gerontol Geriatr. 2014;58(2):278-82.

13. Vasunilashorn S, Coppin AK, Patel KV, Lauretani F, Ferrucci L, Bandinelli S, Guralnik JM. Use of the short physical performance battery score to predict loss of ability to walk 400 meters: analysis from the InCHIANTI study. J Gerontol Ser A. 2009;64A(2):223-9.

14. Fisher S, Ottenbacher KJ, Goodwin JS, Graham J, Ostir GV. Short physical performance battery in hospitalized older adults. Aging Clin Exp Res. 2009; 21(6):445.

15. Nastasi AJ, McAdams-DeMarco MA, Schrack J, Ying H, Olorundare I, Warsame F, Mountford A, Haugen CE, Gonzalez Fernandez M, Norman SP, et al. Pre-kidney transplant lower extremity impairment and post-kidney transplant mortality. Am J Transplant Off J Am Soc Transplant Am Soc Transplant Surg. 2017. 
16. Austin PC, Rothwell DM, Tu JV. A comparison of statistical modeling strategies for analyzing length of stay after CABG surgery. Health Serv Outcomes Res Methodol. 2002;3(2):107-33.

17. Ravangard R, Arab M, Rashidian A, Akbarisari A, Zare A, Zeraati H. Comparison of the results of cox proportional hazards model and parametric models in the study of length of stay in a tertiary teaching Hospital in Tehran, Iran. Acta Med Iran. 2011;49(10):650-8.

18. Verburg IMM, de Keizer NF, de Jonge E, Peek N. Comparison of regression methods for modeling intensive care length of stay. PLoS One. 2014;9(10):e109684.

19. Kramer AA, Zimmerman JE. A predictive model for the early identification of patients at risk for a prolonged intensive care unit length of stay. BMC Med Inform Decis Making. 2010;10:27.

20. Weissman C. Analyzing the impact of long-term patients on ICU bed utilization. Intensive Care Med. 2000;26(9):1319-25.

21. Hemmelgarn BR, Manns BJ, Quan H, Ghali WA. Adapting the Charlson comorbidity index for use in patients with ESRD. Am J Kidney Dis. 2003;42(1):125-32.

22. Cox C, Chu H, Schneider MF, Muñoz A. Parametric survival analysis and taxonomy of hazard functions for the generalized gamma distribution. Stat Med. 2007;26(23):4352-74.

23. McAdams-DeMarco MA, Grams ME, Hall EC, Coresh J, Segev DL. Early hospital readmission after kidney transplantation: patient and center-level associations. Am J Transplant. 2012;12(12):3283-8.

24. McAdams-DeMarco MA, Isaacs K, Darko L, Salter ML, Gupta N, King EA Walston J, Segev DL. Changes in frailty after kidney transplantation. J Am Geriatr Soc. 2015;63(10):2152-7.

25. McAdams-DeMarco MA, James N, Salter ML, Walston J, Segev DL. Trends in kidney transplant outcomes in older adults. J Am Geriatr Soc. 2014;62(12):2235-42.

26. McAdams-DeMarco MA, Suresh S, Law A, Salter ML, Gimenez LF, Jaar BG, Walston JD, Segev DL. Frailty and falls among adult patients undergoing chronic hemodialysis: a prospective cohort study. BMC Nephrol. 2013;14(1):224.

27. McAdams-DeMarco MA, Tan J, Salter ML, Gross A, Meoni LA, Jaar BG, Kao WH, Parekh RS, Segev DL, Sozio SM. Frailty and cognitive function in incident hemodialysis patients. Clin J Amer Soc Nephrol. 2015;10(12):2181-9.

28. McAdams-DeMarco MA, Ying H, Olorundare I, King EA, Haugen C, Buta B, Gross AL, Kalyani R, Desai NM, Dagher NN, et al. Individual frailty components and mortality in kidney transplant recipients. Transplantation. 2016.

29. Exterkate L, Slegtenhorst B, Kelm M, Seyda M, Schuitenmaker J, Quante M, Uehara $\mathrm{H}$, El Khal A, Tullius S, Md P. Frailty and transplantation. Transplantation. 2016;100(4):727-33.

30. Lai JC, Feng S, Terrault NA, Lizaola B, Hayssen H, Covinsky K. Frailty predicts waitlist mortality in liver transplant candidates. Am J Transplant. 2014;14(8): 1870-9.

31. Derck J, Thelen A, Cron D, Friedman J, Gerebics A, Englesbe M, Sonnenday C. Quality of life in liver transplant candidates: frailty is a better Indicator than severity of liver disease. Transplantation. 2015;99(2):340-4.

32. Fried $L P$, Tangen $C M$, Walston J, Newman AB, Hirsch C, Gottdiener J, Seeman T, Tracy R, Kop WJ, Burke G, et al. Frailty in older adults: evidence for a phenotype. J Gerontol A Biol Sci Med Sci. 2001;56(3):M146-56.

33. Ostir GV, Volpato S, Fried LP, Chaves P, Guralnik JM. Reliability and sensitivity to change assessed for a summary measure of lower body function: results from the Women's health and aging study. J Clin Epidemiol. 2002;55(9):916-21.

34. Wang CW, Covinsky KE, Feng S, Hayssen H, Segev DL, Lai JC. Functional impairment in older liver transplantation candidates: from the functional assessment in liver transplantation study. Liver Transpl. 2015;21 (12):1465-70.

35. Lorenz EC, Cheville AL, Amer H, Kotajarvi BR, Stegall MD, Petterson TM, Kremers WK, Cosio FG, LeBrasseur NK. Relationship between pre-transplant physical function and outcomes after kidney transplant. Clin Transpl. 2017: e12952-n/a

36. Guralnik JM, Ferrucci L. Assessing the building blocks of function: utilizing measures of functional limitation. Am J Prev Med. 2003;25(3 Suppl 2):112-21.

37. Verbrugge LM, Jette AM. The disablement process. Soc Sci Med. 1994;38(1):1-14.

38. Kutner NG, Zhang R, Bowles T, Painter P. Pretransplant physical functioning and kidney patients' risk for posttransplantation hospitalization/death: evidence from a national cohort. Clin J Amer Soc Nephrol. 2006; (4):837-43.

39. Cheng XS, Myers JN, Chertow GM, Rabkin R, Chan KN, Chen Y, Tan JC. Prehabilitation for kidney transplant candidates: is it time? Clin Transpl. 2017;31(8).

40. Van Pilsum Rasmussen S, Konel J, Warsame F, Ying H, Buta B, Haugen C, King E, DiBrito S, Varadhan R, Rodriguez-Manas L, et al. Engaging clinicians and patients to assess and improve frailty measurement in adults with end stage renal disease. BMC Nephrol. 2018;19(1):8.

\section{Ready to submit your research? Choose BMC and benefit from}

- fast, convenient online submission

- thorough peer review by experienced researchers in your field

- rapid publication on acceptance

- support for research data, including large and complex data types

- gold Open Access which fosters wider collaboration and increased citations

- maximum visibility for your research: over $100 \mathrm{M}$ website views per year

At BMC, research is always in progress.

Learn more biomedcentral.com/submissions 